Erratum

\title{
Hydrogen Peroxide and Hydroxyl Radical Formation by Methylene Blue in the presence of Ascorbis Acid
}

G. R. Buettner, Th. P. Doherty, and Th. D. Bannister

Department of Chemistry, Wabash College, Crawfordsville, IN 47933, USA

Radiat Environ Biophys (1984) 23:235-243

Due to an unfortunate error the institution address was omitted from the above-mentioned article.

It should read:

Department of Chemistry, Wabash College, Crawfordsville, IN 47933, USA 